\title{
Factors Associated with Occurrence of Peripheral Neuropathy among HIV/AIDS Clients at Kombewa Sub County Hospital of Kisumu County, Kenya
}

\author{
Damian Otieno Opemo \\ School of Nursing, Kibabii University, Bungoma, Kenya \\ Email: opemodamian@gmail.com
}

How to cite this paper: Opemo, D.O. (2020) Factors Associated with Occurrence of Peripheral Neuropathy among HIV/AIDS Clients at Kombewa Sub County Hospital of Kisumu County, Kenya. Open Journal of Nursing, 10, 665-675.

https://doi.org/10.4236/ojn.2020.107047

Received: June 14, 2020

Accepted: July 11, 2020

Published: July 14, 2020

Copyright $\odot 2020$ by author(s) and Scientific Research Publishing Inc. This work is licensed under the Creative Commons Attribution International License (CC BY 4.0).

http://creativecommons.org/licenses/by/4.0/

\section{(c) (i) Open Access}

\begin{abstract}
Peripheral Neuropathy (PN) commonly arises as HIV related nerve complication. The study made attempts to assess the underlying factors leading to peripheral neuropathy, the disabling effects of peripheral neuropathy on a patient's life quality and measures which can be implemented to prevent or lessen the burdening effects of these neuropathies. A descriptive design was adopted where cross-sectional data targeting a sample of 100 consenting adult HIV/AIDS clients were collected utilizing structured questionnaires as key instruments of data collection. In this study variables such as age, height, the past and current CD4 lymphocytes, the viral load, the average duration of antiretroviral treatment (ART), use and the presence of certain co-infection, diabetes or alcohol abuse were analyzed to determine their effects of occurrence of peripheral neuropathy. Fifty eight percent (58\%) of the clients were classified to be having neuropathic as compared $42 \%$ who did not have neuropathic pain. Duration of diagnosis with HIV and previous CD4 count did not significantly determine the occurrence of neuropathic pain. Viral load at time of diagnosis with HIV; nutritional uptake and HAART regimen significantly contribute to occurrence of PN among HIV/AIDS clients. There was no significant association between cases of peripheral neuropathy and demographic factors such as age, gender, height of the respondents and level of education. Clients with peripheral neuropathy were not receiving adequate health talks on peripheral neuropathy. Effect of PN on physical activity was observed. There is need for providing both medical and palliative treatment to HIV/AIDS patients suffering from peripheral neuropathy.
\end{abstract}

\section{Keywords}

Peripheral Neuropathy, HIV/AIDS, Kenya 


\section{Introduction}

\subsection{Study Background}

Countries across the globe have experienced HIV related burden on their economies and health systems, especially in Africa characterized by high prevalence rates. CD4 T-cells are progressively depleted by HIV enhancing the likelihood of opportunistic infections. These infections directly lead to AIDS mortalities and morbidities. Treatment of these infections has evidently minimized mortality rates even before the era of usage for HAART. HIV related mortality has continuously occurred even after ARTs. Many patients who have failed to adhere to drug schedules, have suffered consequences of drug toxicity while others acquired a drug resistant strain of HIV-1. Consequently, morbidity and mortality of patients due to Opportunistic Infections (OI) have continued to occur despite the usage of ART [1].

Among the significant effects of HIV is on neurological wellbeing of a patient. Neurological outcomes of HIV can occur at any time starting at the viral acquisition stage to later phases of AIDS. These occurrences vary and can impact on any part of the nervous system including spinal cord, brain, autonomous nervous system, spinal cord, peripheral nervous system and autonomous nervous system with at least $70 \%-80 \%$ of patients affected by either the virus or OIs. The effect is more pronounced during severe immunodeficiency phase [2].

Peripheral Neuropathy (PN) is one of the most frequent neurological complication linked to human immunodeficiency virus type 1 (HIV) infection together with the advanced acquired immunodeficiency syndrome [3]. PN causes complications across all stages of HIV/AIDS. This can be caused by diverse mechanisms including metabolic, inflammatory, nutritional, toxic and infectious factors. Detecting PN is vital even as many patients utilize nucleoside replicates for treating the HIV disease [4]. The importance to correctly distinguish between intoxication by nucleoside analogues resulting into neuropathies and those related to HIV aids cannot be over emphasized. The neuropathies will cause numbness, pain and weakness. Mononeuropathy is when a single nerve is affected while polyneuropathy is where many or all of the nerves are involved and the symptoms are majorly profound at the extremities of the limbs. Diagnosis and timely treatment of PN can sustain ARV treatment and enhance good health [5]. The identification and treatment of the much overlooked peripheral neuropathies associated with use of nucleoside drugs are reviewed.

Improvement in the life expectancy of HIV/AIDS patients has increased over the past much as the patients still have risks of falling ill due to PN and developing chronic disabilities secondary to those impacted by HIV aids [6] [7]. According to [8], quality of life of persons living with HIV has become increasingly important with the goals of therapy now including improvement of quality of life in addition to reduction of symptoms, suppression of the virus and extension of survival. Peripheral neuropathy is one condition associated with functional limitations which can affect the quality of life of patients. Chronic painful symp- 
toms as experienced with peripheral neuropathy can have a considerable impact on an individual's life and may be associated with anxiety, depressions and loss of mobility and independence [9]. Thus the need for increased and improved surveillance for HIV associated peripheral neuropathy will allow early interventions to improve quality of life and prevent toxicities. It's therefore important to identify the quality of life PLWH in different populations in order to plan and implement effective management of HIV.

In their study in Bamako, Mali realized that neuropathic pains induced by HIV/AIDS are frequent and able to impact negatively the quality of life of patients and their observance of treatment. The individuals with peripheral neuropathy have difficulty in carrying out functions of daily living including difficulty in walking, balancing and working. Although the management of HIV/AIDS in this sub county includes the provision of ARVS and psychological support, it is deemed important to determine whether there are other needs of PLWH which could be addressed. Despite the efforts of countries to reduce the symptoms of HIV/AIDS, suppressing the virus and extension of survival of those infected with HIV, neurologic pain still remains a real public health problem for people living with HIV/AIDS [10].

Therefore the aim of this study is to determine the prevalence of $\mathrm{PN}$ among adults living with HIV/AIDS and quality of life of such people attending outpatient clinic in Kombewa district hospital. These impacts on an individual's life are often associated with anxiety, depression and withdrawal [9].

Many of the clients attending Kombewa Patient Support Centre complicate with peripheral neuropathy end up with walking difficulties, muscle wasting and sometimes poor urine and fecal control. This increases the burden of HIV disease and increased cost of physical rehabilitation thus the need to isolate and minimize the occurrences of peripheral neuropathy in HIV clients.

The study therefore intended to assess the underlying factors leading to peripheral neuropathy, the disabling effects of peripheral neuropathy on the quality of life of the persons affected and measures which can be put in place to prevent or lessen the burdening effects of these neuropathies.

\subsection{Objectives}

The broad objective of this investigation was to examine factors associated with the occurrence of peripheral neuropathy among HIV and AIDS clients at Kombewa Sub County hospital. The objective was achieved through the following specific tasks:

1) Determination of the prevalence of peripheral neuropathy among HIV and AIDS clients attending PSC Kombewa District Hospital;

2) Determination of demographic factors influencing occurrence of peripheral neuropathy among HIV and AIDS clients attending PSC Kombewa District Hospital;

3) Assessment of the measures put in place to prevent/control severity of pe- 
ripheral neuropathy among HIV and AIDS clients attending PSC Kombewa District Hospital;

4) Description of the impact of peripheral neuropathy on quality of life among HIV and AIDS clients attending PSC Kombewa District Hospital.

\section{Methods}

\subsection{Area of Study}

The study facility in which the investigation was carried out is Kombewa Sub County Hospital at the HIV Patient Support Centre (PSC) at sub-county hospital in Seme Sub-County of Kisumu County. The health facility is located a few kilometers off Kisumu-Bondo highway. The institution offers promotive, preventive, diagnostic, curative and rehabilitative health services. It is also involved in medical research in collaboration with other partners that include; WRP, $\mathrm{CDC}$ and KEMRI. It has various departments offering these health care services; General outpatient, $\mathrm{MCH} / \mathrm{FP}$, medical laboratory, radiology, pharmacy, physiotherapy, public health, inpatient and patient support center departments. It has a 40 bed capacity distributed in two wards which usually host between 25 - 60 patients daily. Seme is one of the constituencies of Kisumu County and has a population of 98,805 persons on a 190.2 square kilometer land.

The economic activities of the people around are mainly peasant farming, livestock keeping, and fishing and small scale business. The mean temperature ranges from a minimum of 20 to 35 degrees centigrade. Annual rainfall ranges from 1200 to 1300 per annum. There is considerable variation in weather conditions strongly influenced by the Lake Victoria basin. The area has high variation of temperatures. This contributes to the high rates of food insecurity caused by low agricultural output. The area relies on food supply from neighboring counties. The basic report on well-being in Kenya show that the population is below poverty line [11]. The report reveals that children under 5 years are at risk of either acute or severe malnutrition and that one out of every five children of less than 18 years of age has lost one or both parents mainly due to HIV/AIDS related deaths. The report also indicates that $65.15 \%$ of the hospitals in Kisumu County are public and government owned and that the county has a ratio of one to fifteen thousand doctor populations. 126 out of 10,000 populations in Kisumu have Tuberculosis disease. This is higher than the National count of 39 per 10,000 , Kisumu being ranked 47/47. It is important to note that the HIV prevalence in the county is $12 \%$ higher than the national $6.1 \%$ [12].

\subsection{Study Design}

This was a descriptive cross-sectional study descriptive studies are used to investigate and describe phenomena in real life context. Therefore the design provides a deep understanding of the events being studied and its instruments are helpful in getting firsthand experience as well the ability to allow large amount of data to be collected quickly and at minimal costs. 


\subsection{Study Population}

The study target HIV/AIDS clients attending Kombewa PSC. Eligible clients were adult clients on regular follow up at the PSC. The department was visted by an average of 70 clients per day and operated 5 days a week.

\subsection{Sampling and Sample Size Determination}

Since there was no estimate available of the proportion in target population assumed to have characteristic of peripheral neuropathy among PLWH in Kombewa sub county hospital, convenience sampling was employed in the study targeting 104 respondents. The hospital was an exclusive health facility for HIV positive clients only, were random sampling was employed.

\subsection{Data Collection Instrument and Procedures}

Data on the risk factors contributing to occurrence of peripheral neuropathy was collected using a questionnaire. Questionnaire with both closed and open-ended questions was formulated and used for data collection. The questionnaires were organized into six sections. The first section dealt with the participants' socio-demographic information. The second part had information on past medical history. Section three covered neurological examination using the Tibt test. The fourth section covered practices which favor occurrence of peripheral neuropathy. The fifth section covered assessment of measures used to prevent and control peripheral neuropathy. The sixth section covered effects of peripheral neuropathy on quality of life. Questionnaires were administered in the language best understood by the participant/parent/caretaker, like Luo, Swahili or English. During data collection process the researcher or designee explained the purpose of the study to the participants and sought their permission by way of signing and dating consent form before collecting data. Communication to the respondents was done mainly in English, Kiswahili and Luo.

\subsection{Ethical Considerations}

Permission was obtained from Sub-County Health Management of Kombewa District Hospital. The sampled participants were briefed about study assured of confidentiality and had their individual consent obtained before data collection was initiated.

\section{Results}

This study identified 104 clients with HIV/AIDS. 100 respondents consented to participate in the study, giving $96 \%$ response rate whose findings are as presented in Figures 1-7.

The pie chart above shows the proportion of clients subjected to Tibt test for neuropathic pain. It can be observed that fifty eight percent (58\%) of the clients were reactive to Tibt test, an outcome associated with neuropathic pain compared to $42 \%$ who were observed to be non-reactive. This finding prompted the 


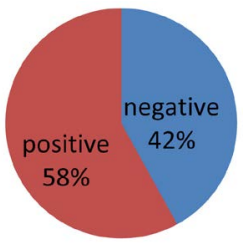

Figure 1. Prevalence of peripheral neuropathy among HIV and AIDS clients.

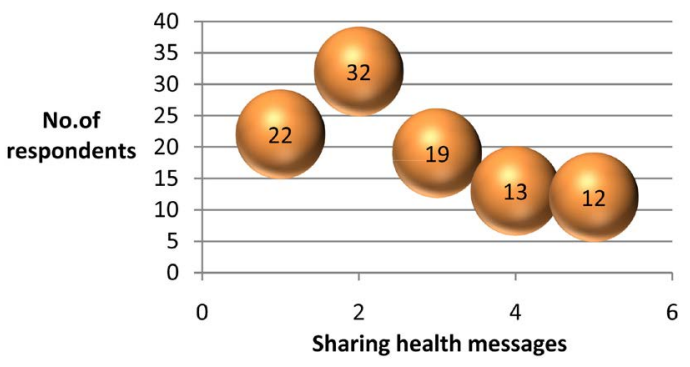

Figure 2. Sharing of health messages on peripheral neuropathy.

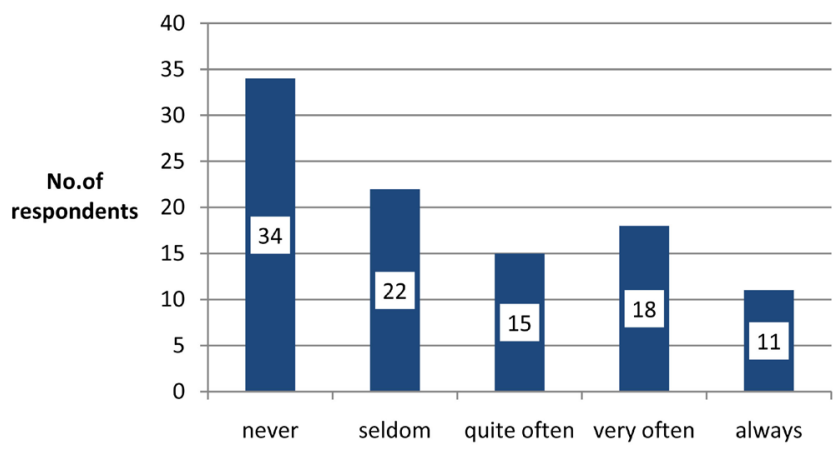

Figure 3. Treatment of peripheral neuropathy.

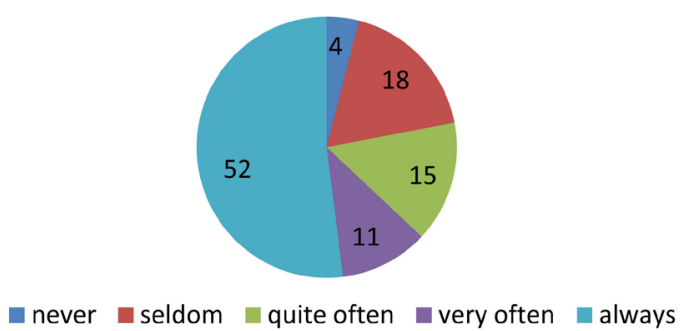

Figure 4. Received nutritional advice on peripheral neuropathy.

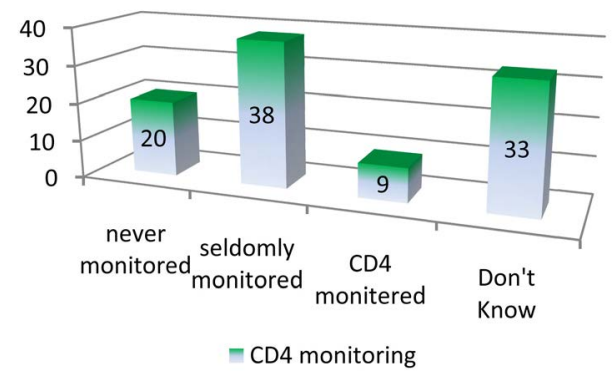

Figure 5. Percentage of respondents who receive CD4 monitoring. 


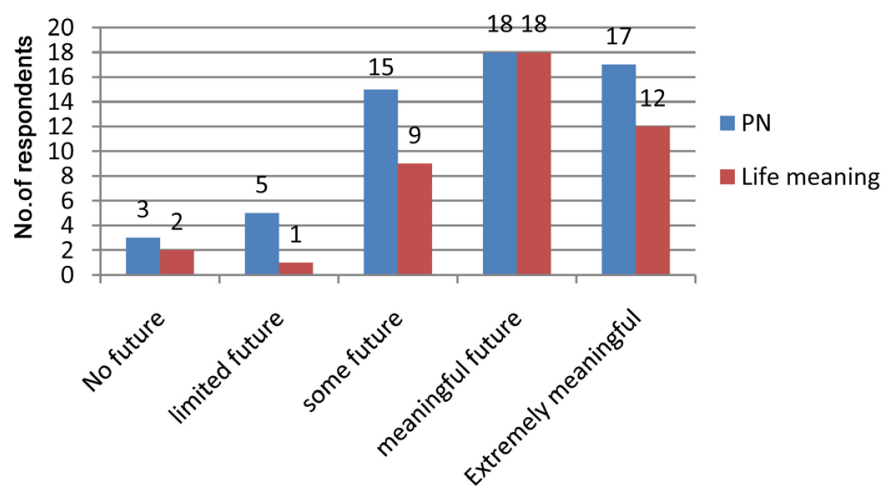

Meaningfulness of life

Figure 6. perception of the value of life by PN patients.

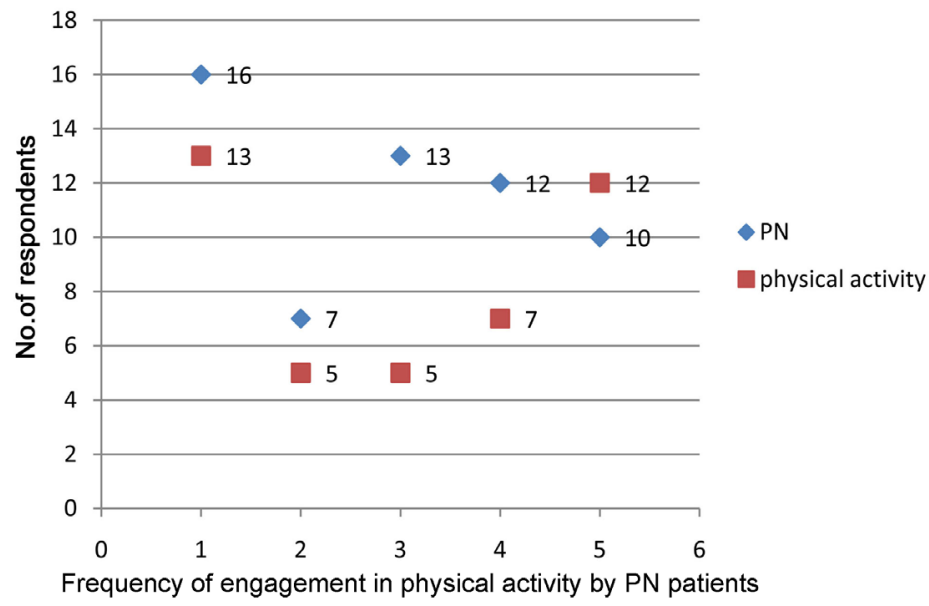

Figure 7. Impact of peripheral neropathy on physical activity.

researcher to assess the impact of past medical history on prevalence of Neuropathic Pain. Time of HIV diagnosis was examined and CD4 count at the time of diagnosis observed. The time when ARV was initiated including the drug combination was also recorded. The results are as shown in Table 1.

The associative relationship between time of diagnosis and CD4 count was observed to be non-significant.

Figure 2 shows the frequency of sharing of health messages on peripheral neuropathy among the respondents. At least 54 percent of the people interviewed rarely participated in sharing health messages on peripheral Neuropathy with 22 percent not sharing at all. At least 44 percent of the respondents shared messages on peripheral neuropathy with $19 \%, 13 \%$ and $12 \%$ sharing quite often, very often, and always respectively. Two percent of the respondents were not sure.

Regarding the treatment of peripheral neuropathy, 34 percent of the respondents never had treatment compared to $22 \%, 15 \%, 18 \%$ and $11 \%$ who were seldomly treated, were treated quite often, treated very often and always treated respectively as shown in Figure 3. 
Table 1. Risk factors contributing to neuropathic pain.

\begin{tabular}{ccccc}
\hline Factor & Category & Yes-NP & No-NP & Pearson Chi-Square \\
\hline \multirow{2}{*}{ Time of Diagnosis } & $>2$ years & 10 & 7 & \\
& $3-5$ years & 24 & 17 & $2 \%=0.143$, \\
& $>6$ years & 24 & 18 & $\mathrm{df}=3, \mathrm{p}=0.986$ \\
& $<200$ & 16 & 13 & \\
CD4 Count & $201-350$ & 17 & 09 & $\#=1.244$, \\
& $351-500$ & 10 & 10 & $\mathrm{~d}=0.743$ \\
& $>500$ & 15 & 10 & \\
Viral load & $<400$ & 53 & 28 & $X^{2}=12.810$ \\
& $<400$ & 2 & 17 & $\mathrm{df}=1$ \\
\hline
\end{tabular}

The pie chart shows the proportion of respondents who were given nutitional advice on peripheral neuropathy. The findings indicate that $52 \%$ never received relevant nutritional advice as compared to $18 \%, 15 \%, 11 \%$ and $4 \%$ who seldomly, quite often, very often and always received nutritional advice on the condition.

Monitoring of CD4 count was an occurance that was examined.20 percent of the respondents were observed to have never considered CD4 monitoring while 38 percent were seldomly exposed to CD4 monitoring. 9\% of the repondents were CD4 monitored while 33\% did not understand CD4 monitoring.

A comparative examination of the perception of respondents regarding the value of life for people with peripheral neuropathy was carried out. It was observed that patients with PN still considered their life to be meaningful despite their illness with 18 percent of the respondents being of the opinion that peripheral neuropathy and the value of life was one and the same thing. Figure 6 illustrates the findings.

The frequency of physical exercise by PN patients was investigated. Figure 7 compares the impact of peripheral neuropathy on physical activity. The results reveal that 16 of the clients indicated PN did not affect their physical activity. On the other hand 10 of the clients with peripheral neuropathy indicated that the condition extremely affected their physical activity. It was also observed that 7\% and $13 \%$ of the clients believed that PN slightly and moderately affected their physical activity respectively.

In general, fifty eight percent (58\%) of the clients were observed to be having neuropathic pain based on Tibt test as compared $42 \%$ who did not have neuropathic pain. Duration of diagnosis with HIV and previous CD4 count did not significantly determine the occurrence of neuropathic pain. On the other hand, viral load at time of diagnosis with HIV significantly contributed to occurrence of peripheral neuropathy. Other risk factors such as taking alcohol, current CD4 count, and duration on HAART did not significantly contribute to occurrence of peripheral neuropathy. Nutritional uptake $(\rho=0.0189)$ and HAART regimen ( $\rho$ 
$=0.01)$ significantly contribute to occurrence of peripheral neuropathy among HIV/AIDS clients. Developing peripheral neuropathy was not significantly related to demographic factors such as Age, height of the respondents, gender and level of education of the respondents who took part in this study.

Regarding patients support measures on managing $\mathrm{PN}$, this study revealed that PN clients were not exposed to adequate health information on their condition. It was observed that clients with peripheral neuropathy rarely received adequate treatment. However it was evident that nutritional advice on peripheral neuropathy was being provided to clients. The study observed that monitoring of CD4 count was not carried out regularly as expected.

The impact of peripheral neuropathy on quality of life was examined and observed to significantly affect the physical activity of patients. The use of analgesics was investigated. The outcome of the investigation revealed that at least one third of clients required daily medication to cope with pain caused by peripheral neuropathy. $85 \%$ of clients interviewed expressed dissatisfaction with level of support being received from family and friends. It was observed that despite having peripheral neuropathy, $35 \%$ of clients had a strongly positive feeling about life while $15 \%$ had moderately positive feelings about life, while $8 \%$ experienced negative feelings about life.

\section{Discussion of Findings}

\section{Prevalence of Peripheral Neuropathy}

The proportion of patients with peripheral neuropathy is high among patients living with HIV/AIDS as realized in this study. Among the HIV patients it was observed that having high viral load predisposes a client to later occurrence of peripheral neuropathy. These findings are consistent with Symth et al., (2007) in a study conducted in Australian outpatient clinic which showed that the prevalence of HIV-sensory neuropathy was high among HIV patients. Similar findings have also been reported in studies conducted at a nearby Jaramogi Oginga Odinga Teaching and Referral Hospital, which showed that the cumulative incidence of peripheral neuropathy stands at 38.2\% among people living with HIV/AIDS [13]. In this regard Wong et al. indicates that HIV positive individuals go through painful sensory neuropathy as such involvement of the peripheral nervous system in HIV infection has been one of the most challenging aspects of the AIDS epidemic. In concurring with findings of this study [10] indicated that there is high frequency of neurological pain during HIV/AIDS patients in Africa and it continues to be a public health problem if no action is taken. Studies by [3] describe peripheral neuropathy as common in individuals infected with the human immunodeficiency virus (HIV). He indicates that peripheral neuropathy may arise as a complication of HIV infection itself, of drug therapy or of other host factors, such as diabetes.

Among the risk factors realized in this study, the viral load, nutritional intake and type of HAART significantly contributed to occurrence of peripheral neuropathy among HIV/AIDS patients/clients. In this regard viral load indicates the 
burden of the disease in the body, viral multiplication and replication in the body significantly affect neurons as viral deposits form on nerve neurons. Consistent with the findings of this study [14] indicated that high viral load significantly contributed to occurrence of peripheral neuropathy among HIV patients in Romania.

In this study it was realized that demographic factors such as age and height of the patients did to significantly contribute to occurrence of peripheral neuropathy in HIV/AIDS patients. This contrasted with findings of [15] which indicated that neuropathy remains a prevalent problem among HIV/AIDS patients of old age. In contrast to the findings of this study Cherry 2009 in his study conducted to determine the demographic factors associated with peripheral neuropathy, and concluded that age and height of the individual play a great role in predicting the development of peripheral neuropathy in patients receiving Stavudine. The results showed that age and height cut offs of more than $170 \mathrm{~cm}$ and 40 years and above had the higher chances of developing neuropathy.

This study showed that peripheral neuropathy is a common health problem among patients living with HIV/AIDS. This study evaluated a number of risk factors and it was evident that high viral load, nutritional status and type of ARV regimen significantly contributed to occurrence of peripheral neuropathy. The results suggest that there is increased risk when there is delayed initiation of ARVs to suppress viral replication.

The present study reveals that there is need for providing both medical and palliative treatment to HIV/AIDS patients suffering from peripheral neuropathy. Consistent with other studies the findings of this study suggest the need peripheral neuropathy impacts on the quality of life of patients as such efforts should be directed at improving their coping ability. The limitations of this study include its cross-sectional nature. The results of this study could be biased given that majority of the patients were recently diagnosed and initiated on ART; hence there could be delayed onset of peripheral neuropathy associated with commitant medication use. It is important to state here that the non-significant $\mathrm{p}$ values although not significant, cannot rule out potentially large associations.

\section{Recommendations}

From the findings of this study a number of recommendations are suggested.

The need to provide consistent health education to patients living with HIV/AIDS on the potential risk factors that would expose them to peripheral neuropathy was established. As realized in this study nutritional advice on proper diet that would help alleviate suffering from peripheral neuropathy. It is recommended that health workers in the Patient Support Centre need to consistently monitor CD4 levels of patients and viral loads as they are the indicators that would help identify those at risk of developing peripheral neuropath.

\section{Conflicts of Interest}

The author declares no conflicts of interest regarding the publication of this paper. 


\section{References}

[1] Solomon, F.B., Angore, B.N., Koyra, H.C., Tufa, E.G., Berheto, T.M. and Admasu, M. (2018) Spectrum of Opportunistic Infections and Associated Factors among People Living with HIV/AIDS in the Era of Highly Active Anti-Retroviral Treatment in Dawro Zone Hospital: A Retrospective Study. BMC Research Notes, 11, 604. https://doi.org/10.1186/s13104-018-3707-9

[2] Centner, C.M., Bateman, K.J. and Heckmann, J.M. (2013) Manifestations of HIV Infection in the Peripheral Nervous System. The Lancet Neurology, 12, 295-309. https://doi.org/10.1016/S1474-4422(13)70002-4

[3] Verma, S., Estanislao, L. and Simpson, D. (2005) HIV-Associated Neuropathic Pain. CNS Drugs, 19, 325-334. https://doi.org/10.2165/00023210-200519040-00005

[4] Simpson, D.M. and Tagliati, M. (1995) Nucleoside Analogue-Associated Peripheral Neuropathy in Human Immunodeficiency Virus Infection. Journal of Acquired Immune Deficiency Syndromes and Human Retrovirology: Official Publication of the International Retrovirology Association, 9, 153-161.

[5] Robinson-Papp, J. and Simpson, D.M. (2008) Neuromuscular Complications of Human Immunodeficiency Virus Infection. Physical Medicine and Rehabilitation Clinics of North America, 19, 81-96. https://doi.org/10.1016/j.pmr.2007.10.009

[6] Demmer, C. (2001) Quality of Life and Risk Perception among Predominantly Heterosexual, Minority Individuals with HIV/AIDS. AIDS Patient Care and STDs, 15, 481-489. https://doi.org/10.1089/108729101753145475

[7] Gale, J. (2003) Physiotherapy Intervention in Two People with HIV or AIDS-Related Peripheral Neuropathy: Case Report. Physiotherapy Research International, 8, 200-209. https://doi.org/10.1002/pri.290

[8] Casado, A. (2005) Measurement of Quality of Life of HIV Individuals: Perspectives \& Future Directions. Indian Journal of Medical Research, 122, 282.

[9] Biraguma, J. and Rhoda, A. (2012) Peripheral Neuropathy and Quality of Life of Adults Living with HIV/AIDS in the Rulindo District of Rwanda. SAHARA-J: Journal of Social Aspects of HIVI AIDS, 9, 88-94.

[10] Maiga, A.I., Fofana, D.B., Maiga, A.C., Diallo, F., Ait-Arkoub, Z., Daou, F., Katlama, C., et al. (2013) Transmitted Antiretroviral Drug Resistance in Newly HIV-Infected and Untreated Patients in Segou and Bamako, Mali. AIDS Research and Human Retroviruses, 29, 182-186. https://doi.org/10.1089/aid.2012.0118

[11] Oyombo, J.O. (2011) Influence of Community Based Organizations' Activities on Community Livelihood Sustainability: A Case of Kombewa Division, Kisumu West District, Kenya. Doctoral Dissertation, University of Nairobi, Nairobi.

[12] NACC (2018) National Aids Control Council. Kenya HIV Estimates Report 2018.

[13] Ndakala, F.N., Oyugi, J.O., Oluka, M.N.W., Kimani, J., Jablonka, A. and Behrens, G.M. (2016) Prevalent Neuropathy in a Cohort of HIV-Infected Kenyan Sex Workers Using Antiretroviral Drugs. Pan African Medical Journal, 25, 14. https://doi.org/10.11604/pamj.2016.25.14.9699

[14] Giubelan, L.I., Cupşa, A., Dumitrescu, F., Niculescu, I. and Stoian, A.C. (2014) Considerations about Risk Factors for Peripheral Neuropathies in Romanian HIV-Infected Patients. Current Health Sciences Journal, 40, 42.

[15] Smyth, K., Affandi, J.S., McArthur, J.C., Bowtell-Harris, C., Mijch, A.M., Watson, K., Cherry, C.L., et al. (2007) Prevalence of and Risk Factors for HIV-Associated Neuropathy in Melbourne, Australia 1993-2006. HIV Medicine, 8, 367-373. https://doi.org/10.1111/j.1468-1293.2007.00478.x 